IX Congresso Brasileiro de Informática na Educação (CBIE 2020)

Anais do XXXI Simpósio Brasileiro de Informática na Educação (SBIE 2020)

\title{
Adaptações em atividades de Pensamento Computacional para estimulação cognitiva em idosos
}

\section{Daniel Araújo de Lucena ${ }^{1}$, Isabel Dillmann Nunes ${ }^{2}$, Rivanilson da Silva Rodrigues ${ }^{1}$, Danyel Rocha de Oliveira Souza ${ }^{3}$}

\author{
${ }^{1}$ Faculdade de Filosofia, Ciências e Letras de Cajazeiras \\ Cajazeiras - PB - Brasil \\ ${ }^{2}$ Instituto Metrópole Digital - Universidade Federal do Rio Grande do Norte \\ Natal - RN - Brasil \\ ${ }^{3}$ UNIFIP - Centro Universitário \\ Patos - PB - Brasil \\ falarcomdanielaraujo@gmail.com, bel@imd.ufrn.br, rivanilson@gmail.com, \\ danyelrocha. souza@gmail.com
}

\begin{abstract}
The aging process is a process that usually causes loss of cognitive perception, but there is a social resource for those who are offered conditions to remain cognitively healthy, even in senescence. This article is directed to Computational Thinking for Cognitive Stimulation through Unplugged Activities that have been Adapted to the Elderly Public. From a study of cognitive stimulation, we found that it is necessary to intervene in activities disconnected from Computational Thinking, statistically produced an improvement in superior performance and cognitive training developed by a psychologist.
\end{abstract}

Resumo. $O$ envelhecimento é um processo que geralmente conduz a perda de capacidades cognitivas, entretanto há um apelo social para que sejam oferecidas condições de manter-se cognitivamente saudável, mesmo na senescência. Este artigo conecta o Pensamento Computacional à estimulação cognitiva por meio de atividades desplugadas que foram adaptadas ao público idoso. A partir de uma pesquisa de estimulação cognitiva, constatou-se que a intervenção baseada em atividades desplugadas de Pensamento Computacional produziu estatisticamente uma melhoria de desempenho superior ao treino cognitivo elaborado por um psicólogo.

\section{Introdução}

Ao longo do envelhecimento pode ocorrer uma redução do desempenho cognitivo, comprometendo, por exemplo, as funções executivas [Cardoso, Landenberger e Argimon 2017]. Na vida diária, os danos às funções executivas resultam em dificuldades no desempenho de atividades comuns como dirigir um carro, no manejo financeiro e no cumprimento de obrigações, levando à redução no número de relações sociais, à depressão e por fỉm, ao isolamento [Assis et al. 2015]. Esse isolamento social leva à perda da identidade, de liberdade, de autoestima, favorecendo muitas vezes a renegar a própria vida, justificando a alta prevalência de doenças mentais [Bertoldi, Batista e Ruzanowsky 2015].

Se o declínio cognitivo, otimizado sobretudo na senescência, é capaz de comprometer a capacidade de resolução de problemas, isso impacta negativamente na possibilidade do idoso 
IX Congresso Brasileiro de Informática na Educação (CBIE 2020)

Anais do XXXI Simpósio Brasileiro de Informática na Educação (SBIE 2020)

permanecer empregado, indo na contramão do atual cenário brasileiro onde essa faixa da população vem adiando a saída do mundo do trabalho, passando de 6,3\% em 2012 para 7,8\% em 2018, segundo o Instituto de Pesquisa Econômica Aplicada [IPEA 2018].

Sabe-se que o trabalho é fonte de renda, satisfação pessoal e importante para a manutenção da qualidade de vida [Paolini 2016], entretanto esta escolha do idoso em permanecer empregado é também reflexo da atual conjuntura do país, em que pelo menos 10,8 milhões de brasileiros dependem da renda de idosos aposentados para viver. Só no último ano, o número de residências em que mais de $75 \%$ da renda vem de aposentadorias cresceu $12 \%$, de 5,1 milhões para 5,7 milhões [Brandão 2018].

Portanto deve-se considerar as múltiplas consequências do declínio cognitivo para o idoso, necessitando assim que se encontrem estratégias para a manutenção e melhora das habilidades cognitivas [Cavalli et al. 2017], sobretudo aquelas que são cobiçadas pelo mercado de trabalho, a exemplo da resolução de problemas.

Evidências [Schaie e Willis 1986] têm mostrado que pelo menos parte da perda de funções no envelhecimento pode ser reversível, por meio de técnicas de intervenção como o treino cognitivo, que é capaz de induzir a plasticidade existente no sistema nervoso e, consequentemente, produzir um impacto positivo na qualidade de vida dos idosos.

No que diz respeito ao fomento da capacidade de resolução de problemas, uma alternativa é instruir o idoso ao Pensamento Computacional, que a partir dos seus quatro pilares (decomposição, reconhecimento de padrões, abstração e algoritmo), é uma metodologia de resolver problemas [Wing 2006]. Através de atividades desplugadas, onde a aprendizagem independe de recursos de hardware e software [Bell, Witten e Fellows 2011], o indivíduo com mais de 60 anos passa a utilizar conceitos da Ciência da Computação para construir um processo de pensamento lógico e criativo para resolver problemas [Wing 2014].

Pelo que foi apresentado, percebe-se que o Pensamento Computacional estimula o desenvolvimento de habilidades em seus participantes. No entanto, durante a produção desta pesquisa, percebeu-se a escassez de estudos que conectam o Pensamento Computacional ao público idoso. Investigou-se também possíveis orientações da $\mathrm{SBC}^{1}$ (Sociedade Brasileira de Computação) com relação à aplicação do Pensamento Computacional junto a população longeva, mas não foram encontradas.

Para amenizar essa lacuna, 24 atividades desplugadas de Pensamento Computacional foram selecionadas para formar um instrumento de estimulação cognitiva para idosos. As questões foram extraídas das teses de Brackmann (2017) e de González (2016), e do desafio Bebras $^{2}$, todas elas passando por adaptações, cuja descrição é o objetivo deste trabalho. $\mathrm{O}$ instrumento foi aplicado em um grupo de idosos e constatou-se uma melhora estatística significativa no desempenho dos participantes.

O artigo está divido em 7 seções, das quais se faz presente esta introdução; as adaptações realizadas nas questões selecionadas para compor o instrumento de estimulação cognitiva; uma sessão na qual se discorre sobre o teste de estimulação realizado junto aos idosos; na quarta seção são apresentados os resultados do teste de estimulação; em seguida é apresentado as ameaças à validade e limitações do estudo; logo após denota-se as conclusões e por último, as referências bibliográfica.

\section{Adaptações realizadas}

A pesquisa de Cachioni et al. (2015) enfatiza que a aprendizagem do idoso deve ser significativa, ou seja, é necessário considerar as experiências e necessidades para que essa parcela da população continue a aprender com eficácia. Diante desta condição o instrumento de

${ }^{1}$ https://www.sbc.org.br/

2https://www.bebras.org/ 
IX Congresso Brasileiro de Informática na Educação (CBIE 2020)

Anais do XXXI Simpósio Brasileiro de Informática na Educação (SBIE 2020)

estimulação cognitiva foi elaborado a partir de adaptações realizadas nas 24 questões, destas, 4 foram extraídas de Brackmann (2017), 3 de Gonzáles (2016) e 14 do desafio Bebras.

Em Brackmann (2017) encontra-se um conjunto de 10 atividades baseadas nos pilares do Pensamento Computacional que foram aplicadas com crianças da educação básica da Espanha e do Brasil. A Figura 1 demonstra o primeiro exercício utilizado pelo autor.

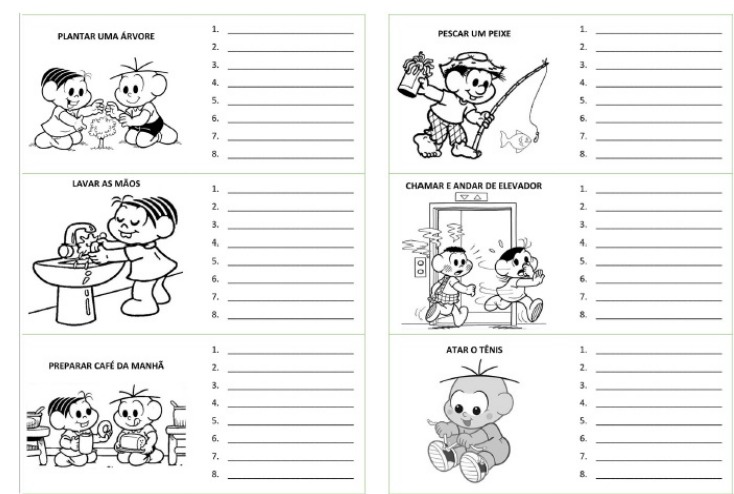

Figura 1: Primeira questão aplicada por Brackmann (2017)

A atividade instiga a criação de listas de instruções necessárias para atingir 6 (seis) objetivos comuns do cotidiano, exercitando três pilares do Pensamento Computacional: abstração, decomposição e algoritmos. As adaptações para o público idoso consistiram em eliminar expressões e personagens infantis, adicionando fotos reais de tarefas do cotidiano. $\mathrm{O}$ resultado é demonstrado na Figura 2.

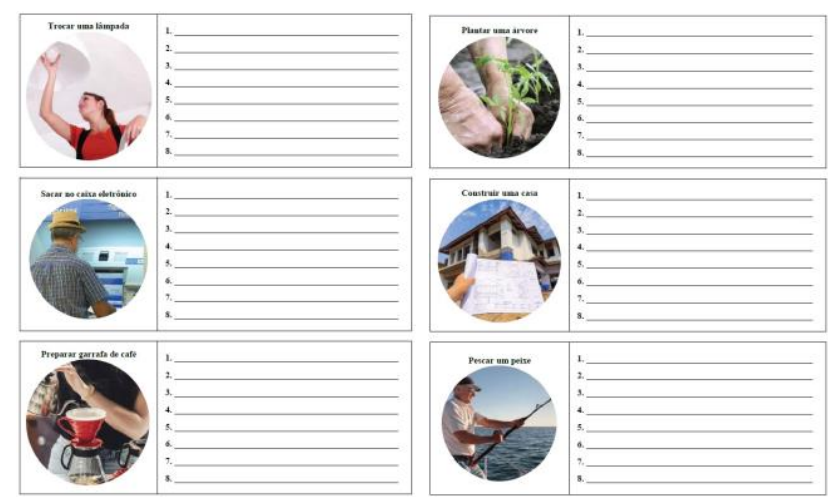

Figura 2: Questão de Brackmann (2017) adaptada para o público idoso

Em González (2016) encontra-se o Teste de Pensamento Computacional composto por 28 atividades. O instrumento busca identificar a habilidade de formação e solução de problemas, utilizando sintaxes lógicas usadas nas linguagens de programação e conceitos dos quatro pilares do Pensamento Computacional. A Figura 3 apresenta uma questão utilizada no teste, onde se pergunta quais são os comandos que levam o 'Pac-Man' até o fantasma pelo caminho indicado. A alternativa correta é a letra $\mathrm{C}$. 
IX Congresso Brasileiro de Informática na Educação (CBIE 2020)

Anais do XXXI Simpósio Brasileiro de Informática na Educação (SBIE 2020)

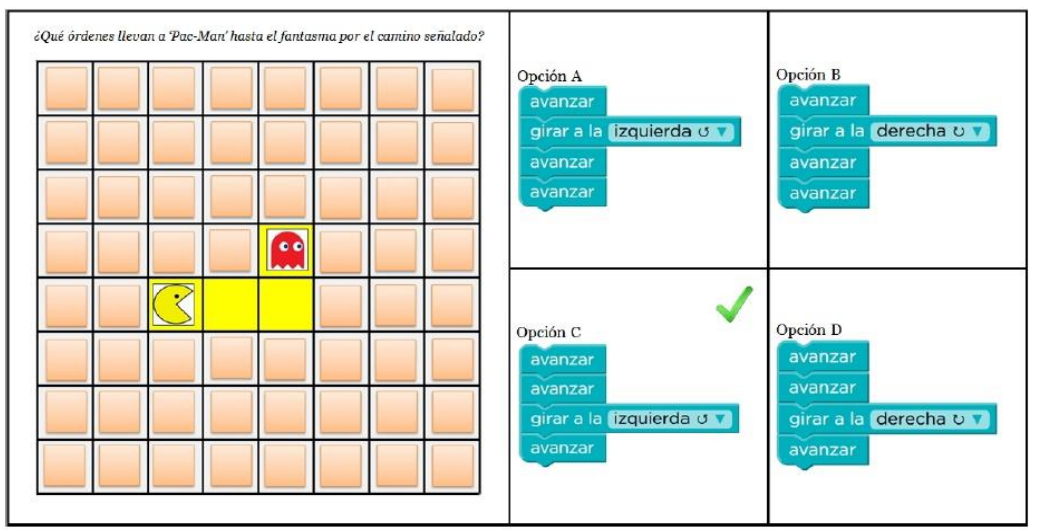

Figura 3: Questão aplicada por Gonzáles (2016)

Na versão adaptada para os idosos, retirou-se os desenhos do "Pac-Man" e do fantasma, substituindo-os pela figura de um indivíduo que deve ser conduzido até seu automóvel, como mostra a Figura 4.

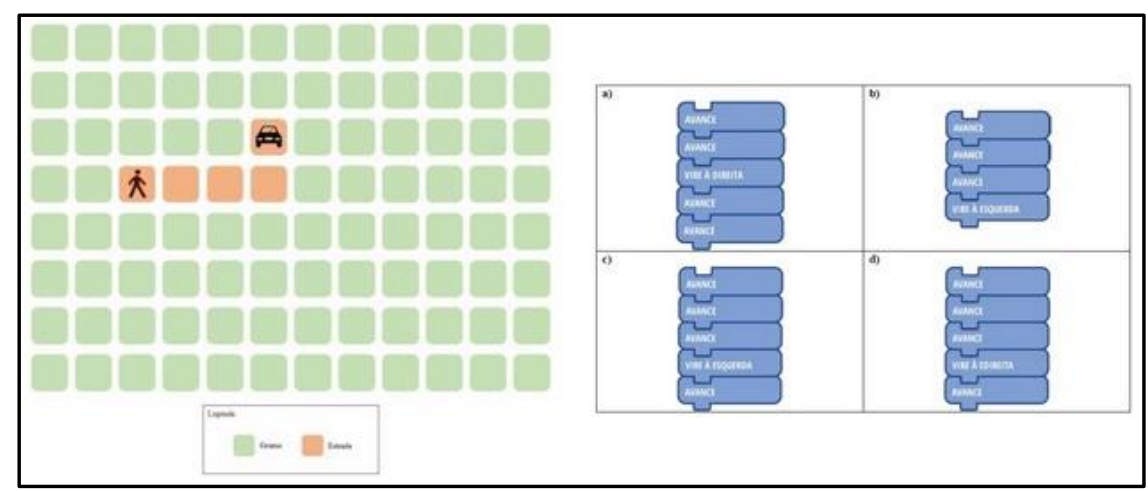

Figura 4: Questão de Gonzáles (2016) adaptada para o público idoso

A terceira fonte de extração de atividades foi o desafio Bebras, uma iniciativa internacional para promover a informática e o Pensamento Computacional entre estudantes de 5 a 19 anos. O desafio Bebras é organizado anualmente por cada um dos 67 países participantes, os quais selecionam as questões que serão aplicadas com seus estudantes, porém, as atividades escolhidas são de uma base de dados comum atualizada anualmente pelo próprio Bebras. A Figura 5 apresenta uma questão original, selecionada do desafio Bebras de 2016 aplicada no Reino Unido, que solicita ao aluno o conjunto de instruções que leva o castor (animal roedor semi-aquático) até a escola através de um carro autônomo.

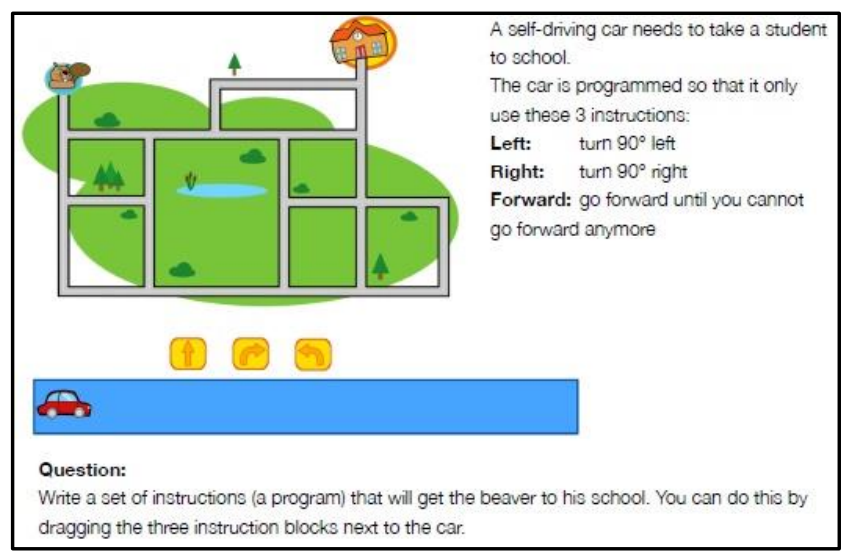

Figura 5: Questão do Desafio Bebras aplicada no Reino Unido em 2016

Na versão traduzida e adaptada, como mostra a Figura 6, optou-se por substituir o castor 
IX Congresso Brasileiro de Informática na Educação (CBIE 2020)

Anais do XXXI Simpósio Brasileiro de Informática na Educação (SBIE 2020)

por um personagem chamado Raimundo e que, ao invés de ir para a escola, desejava chegar em casa. Outros ajustes estão no redesenho do mapa e no fato de que o enunciado afirma que o Raimundo não preferiu pegar um táxi ou motorista de aplicativo, mas escolheu ir num carro autônomo (elemento preservado da questão original). Adaptações equivalentes também ocorreram nas questões extraídas de Brackmann (2017) e González (2016). Um documento ${ }^{3}$ foi criado para apresentar as modificações realizadas em todas as questões. Um segundo arquivo ${ }^{4}$ traz a intervenção cognitiva completa baseada em atividades desplugadas de Pensamento Computacional para idosos.

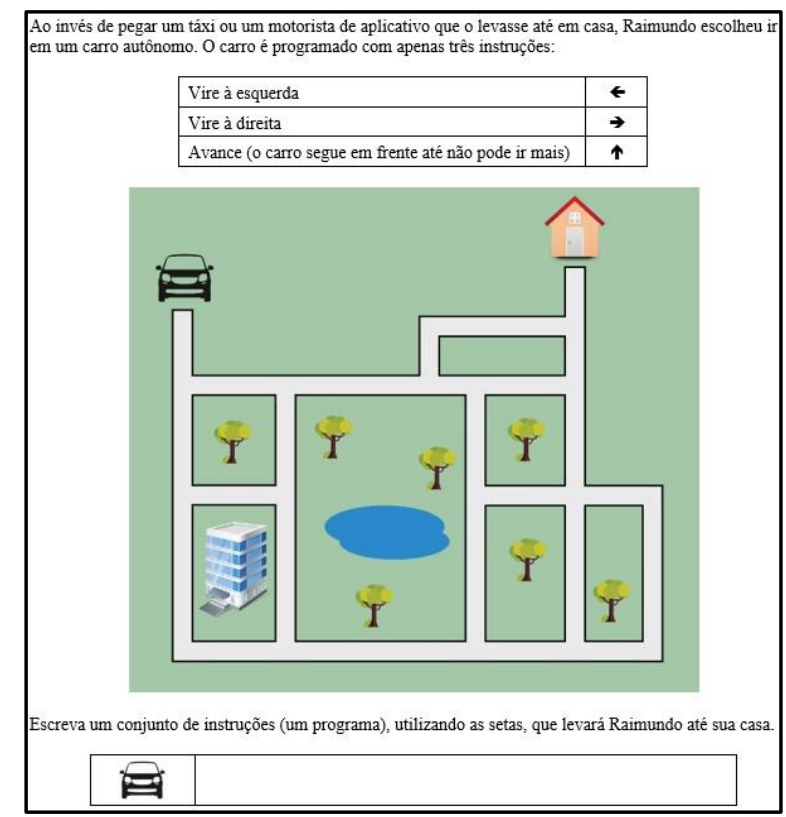

Figura 6: Questão adaptada do Desafio Bebras aplicada no Reino Unido em 2016

Outros ajustes estão no redesenho do mapa e no fato de que o enunciado afirma que o Raimundo não preferiu pegar um táxi ou motorista de aplicativo, mas escolheu ir num carro autônomo (elemento preservado da questão original).

\section{O teste de estimulação cognitiva}

As 24 questões de Pensamento Computacional adaptadas foram aplicadas numa pesquisa de estimulação cognitiva constituída de dois grupos (controle e experimento) de idosos. Ambos os grupos foram submetidos a testes estatísticos com o objetivo de identificar mudança de desempenho, presumindo portanto que a pontuação dos testes realizados após a intervenção sejam superiores aos testes realizados antes.

Como parâmetro a fim de testá-las, foram comparadas as notas dos participantes antes e após o tratamento. Ao término das verificações nos grupos individualmente, ambos foram comparados quanto a mudança de desempenho. A Figura 7 resume a sequência das investigações.

\footnotetext{
${ }^{3}$ https://docs.google.com/document/d/15GYERo1I83gMf-xqtluPrgTLduCWj6j1fRVu-MSlobk/edit?usp=sharing ${ }^{4}$ https://docs.google.com/document/d/1pGWMhd9PlOuVmIEo1SDZq1BNKeC0qQHBAb2TVBghNh4/edit?usp=sharing
} 
IX Congresso Brasileiro de Informática na Educação (CBIE 2020)

Anais do XXXI Simpósio Brasileiro de Informática na Educação (SBIE 2020)

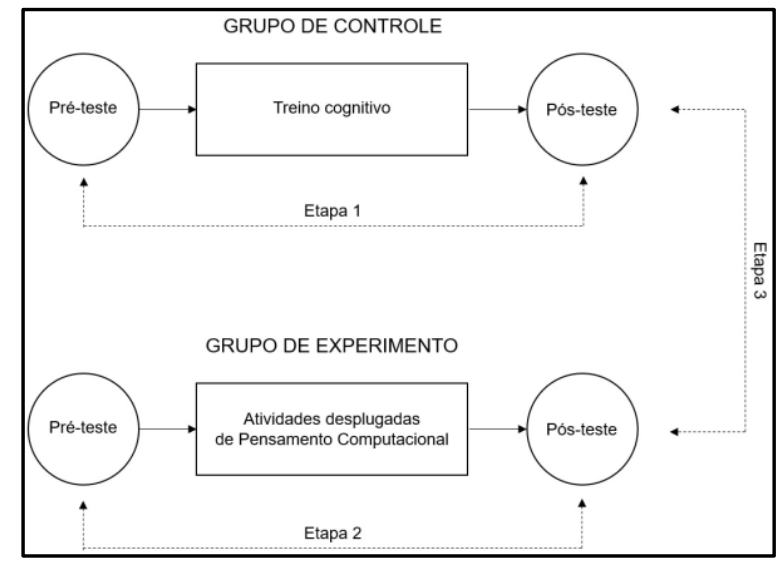

Figura 7: Grupos de idosos

A etapa 1 busca identificar estatisticamente uma melhoria cognitiva nos participantes após o treino cognitivo. Assim também a etapa 2 almeja o mesmo resultado, porém através de atividades desplugadas de Pensamento Computacional. A etapa 3 compara as mudanças de desempenho de ambos os grupos. Vale ressaltar que treinos cognitivos já são utilizados para estimular a cognição em idosos. Não é foco deste trabalho gerar dúvidas sobre a efetividade destes instrumentos já estabelecidos na Psicologia. Busca-se portanto, observar estatisticamente o efeito das sessões baseadas em atividades desplugadas de Pensamento Computacional.

\section{Resultado}

Após a execução de testes estatísticos, o grupo de controle atingiu uma média amostral de 46 com desvio padrão de 3,48 e no pós-teste apresenta média amostral de 45 com desvio padrão de 2,17 . No entanto, o grupo experimental, obteve média amostral de 41,5 com desvio padrão de 2,75 e no pós-teste apresenta média amostral de 46 com desvio padrão de 1,89. Os dados descritos estão na Tabela 1. As demonstrações gráficas que apresentam a melhoria significativa do grupos experimental encontram-se no Gráfico 1 e Gráfico 2.

Tabela 1: Resultados gerais

\begin{tabular}{|c|c|c|c|c|c|}
\hline \multicolumn{6}{|c|}{ CONTROLE } \\
\hline & $\mathrm{N}$ & $\begin{array}{c}\text { Pré-teste } \\
\text { Média (desvio padrão) }\end{array}$ & $\begin{array}{c}\text { Pré-teste } \\
\text { Média (desvio padrão) }\end{array}$ & p-valor & $\begin{array}{c}\text { Alteração do } \\
\text { desempenho }(\%)\end{array}$ \\
\hline Desempenho & 9 & $46(3,48)$ & $45(2,17)$ & 0,96 & $-1,09$ \\
\hline \multicolumn{6}{|c|}{ EXPERIMENTO } \\
\hline & $\mathrm{N}$ & $\begin{array}{c}\text { Pré-teste } \\
\text { Média (desvio padrão) }\end{array}$ & $\begin{array}{c}\text { Pós-teste } \\
\text { Média (desvio padrão) }\end{array}$ & p-valor & $\begin{array}{c}\text { Alteração do } \\
\text { desempenho }(\%)\end{array}$ \\
\hline Desempenho & 7 & $41,5(2,75)$ & $46(1,89)$ & 0,03 & 10,84 \\
\hline
\end{tabular}

A coluna "p-valor" apresenta os resultados do teste de Wilcoxon [HINES et al. 2006] e t Student [Field 2009] para o grupo de controle e experimental respectivamente. Recorda-se que tais testes são utilizados para verificar se há diferença entre as médias do pré e pós-teste. Como no grupo de controle a condição p-valor $<0,05$ não é satisfeita, pode-se afirmar que estatisticamente não há diferença entre as médias do pré e pós-teste, isto é não houve melhoria de desempenho significativo. Já no grupo de experimento, a condição p-valor < 0,05 é satisfeita, 
IX Congresso Brasileiro de Informática na Educação (CBIE 2020)

Anais do XXXI Simpósio Brasileiro de Informática na Educação (SBIE 2020)

afirma-se que estatisticamente há diferença entre as médias do pré e pós-teste.

Sendo assim, a partir das médias e dos p-valores calculados, comprova-se que houve uma melhoria de desempenho significativa apenas no grupo experimental, sendo um indício da eficácia da intervenção cognitiva baseada em atividades desplugadas de Pensamento Computacional.
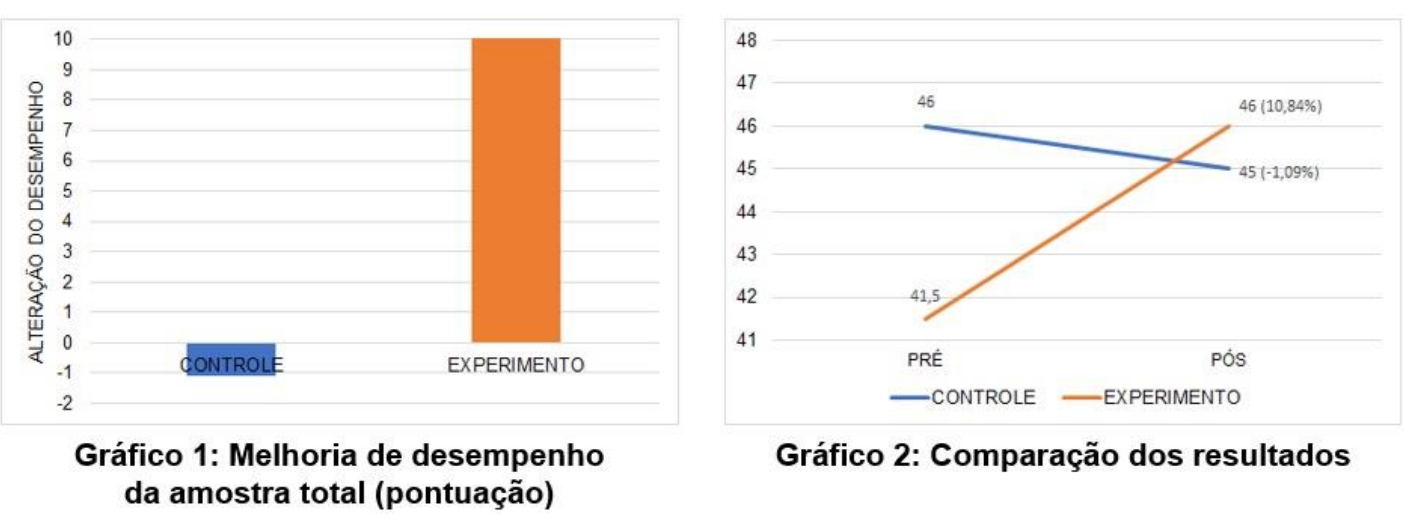

Gráfico 2: Comparação dos resultados

Os resultados alcançados não se configuram como um indicador definitivo para apresentar as sessões desplugadas de Pensamento Computacional como instrumento de enriquecimento cognitivo em idosos, sobretudo por conta das ameaças à validade desta pesquisa, as quais são comentadas na próxima seção. Assim também os indícios gerados nesta investigação não contestam a eficácia dos treinos cognitivos formulados pelos psicólogos. A par desses resultados, o presente estudo busca ser um ponto de partida ao desenvolvimento de novas pesquisas sobre o uso do Pensamento Computacional na senescência.

\section{Ameaças à validade e limitações do estudo}

Neste estudo, alguns dos problemas estão relacionados ao tamanho e a homogeneidade da amostra. A quantidade de participantes (9, para o grupo de controle; 7 para o grupo de experimento) não é ideal do ponto de vista estatístico, gerando limitações nos resultados, sendo estes considerados indícios e não conclusivos com o intuito de se fazer generalizações sobre a população. O obstáculo da homogeneidade caracteriza-se porque os participantes são do mesmo sexo (feminino) e membros de um mesmo projeto de extensão acadêmica.

Outro aspecto a ser considerado é não confundir a significância estatística com a significância clínica. O presente trabalho verifica possíveis melhorias estatísticas no funcionamento cognitivo, pois aferir os efeitos clínicos de uma intervenção requer a análise de profissionais de diferentes áreas. Também está fora do escopo desta pesquisa discutir sobre o tempo de duração das possíveis melhorias cognitivas, assim como atestar que elas ajudarão os participantes nas tarefas cotidianas.

Ficou evidente a necessidade de criar questões de Pensamento Computacional que contemplem atividades cotidianas dos idosos para que se estimule o engajamento dos mesmos durante as sessões. Exercícios que apresentavam blocos de instrução foram consideradas difíceis.

\section{Considerações finais}

Pesquisas vêm sendo empreendidas com o intuito de estudar o funcionamento do cérebro humano. De um lado, há um grupo de pesquisadores que se posiciona de maneira bastante cética e contrária à possibilidade de modificação ou intervenção frente aos possíveis danos cognitivos. Por outro lado, o grupo de pesquisadores favorável à hipótese do enriquecimento cognitivo vem ancorando seus argumentos em evidências como a 
IX Congresso Brasileiro de Informática na Educação (CBIE 2020)

Anais do XXXI Simpósio Brasileiro de Informática na Educação (SBIE 2020)

neuroplasticidade na idade adulta [Santos 2015].

Tendo em vista a possibilidade de melhora cognitiva, o presente trabalho propôs o uso de atividades desplugadas de Pensamento Computacional como alternativa de estimulação nos idosos. Temos dois aspectos que tornam esse trabalho expressivo: 1) considerar o Pensamento Computacional como forma de estimulação cognitiva; 2) correlacionar o Pensamento Computacional com idosos. Adotar o Pensamento Computacional é uma estratégia eficaz para modelar soluções e resolver problemas de maneira eficiente. Tal característica, inclusive, é considerada uma das mais importantes, não apenas para o mundo de trabalho, mas para a vida moderna. A originalidade da pesquisa foi reconhecida pelo DesafIE! ${ }^{5}$, através de uma publicação ${ }^{6}$ em seus anais de 2019.

Reconhecemos que se torna necessário o desenvolvimento de materiais e abordagens no ensino do Pensamento Computacional próprias para idosos, exigindo que profissionais de diferentes áreas atuem com o propósito de criar novas alternativas para desenvolvimento cognitivo para essa faixa da população. Na etapa seguinte, propõe-se a oferta de disciplinas voltadas para a Computação de forma opcional, possibilitando o acesso dos estudantes aos conceitos do Pensamento Computacional para que, futuramente, essas disciplinas façam parte do currículo obrigatório.

O presente estudo sugere que atividades desplugadas de Pensamento Computacional produzem uma melhora de desempenho estatisticamente superior ao treino cognitivo elaborado pelo psicólogo colaborador. Porém, considera-se importante salientar que atividades desplugadas não devem ser entendidas como uma solução completa para a estimulação cognitiva. As atividades desplugadas, a partir de um certo ponto, podem não ser tão eficazes quanto esperado, por manter os idosos distantes de experiências com as tecnologias digitais.

A atual pesquisa elaborou sessões de atividades desplugadas de Pensamento Computacional e avaliou a eficácia das mesmas como método para o desenvolvimento cognitivo de idosos, atestando uma melhora estatística do desempenho dos participantes após a intervenção. É perceptível o esforço para tornar o Pensamento Computacional presente no cotidiano da vida escolar e acadêmica. Ações como a produção de artigos e livros que discutem o currículo da educação formal; formações e palestras para aperfeiçoar metodologias de ensino e propor alternativas na formação de professores; adoção à Base Nacional Curricular Comum etc. É tímida, todavia, as ações que visam correlacionar o Pensamento Computacional com idosos, e mais difícil ainda, apresentá-lo como alternativa de estimulação cognitiva à faixa senescente da população.

No tocante à condução das sessões observa-se a necessidade de uma formação para atuar com a terceira idade, pois é perceptível a tendência de transpor métodos utilizadas nos cursos de graduação, pós-graduação ou do $1^{\circ}$ e $2^{\circ}$ graus. Além disso, a inadequação de materiais pode favorecer a evasão [Alves 1997]. A experiência gerada neste estudo permite compreender que instrumentos, cursos ou demais ações devem ser elaboradas junto com os idosos, ou seja, devem partir de um levantamento das suas necessidades, sob pena de ser pouco significativa para eles [Martinez 1998]. Assim, as ações que têm os idosos como público, devem tê-los como parceiros na elaboração de conteúdos e materiais [Giubilei 1993].

Apoiados no princípio da educação permanente, deve-se compreender que a busca dos idosos é a fruição, o gosto por aprender, a realização de sonhos e projetos de vida adiados, a necessidade de se sentir vivo, ativo, atualizado e inserido na sua comunidade [Cachioni 2015]. Portanto, é imprescindível que as atividades educacionais para a terceira idade devam ter como ponto de partida conhecimentos específicos sobre as características dessa parcela da população, que possui peculiaridades garantidas pelo seu próprio desenvolvimento e experiências

${ }^{5}$ https://www.br-ie.org/pub/index.php/desafie/index

${ }^{6}$ https://www.br-ie.org/pub/index.php/desafie/article/view/8789 
IX Congresso Brasileiro de Informática na Educação (CBIE 2020)

Anais do XXXI Simpósio Brasileiro de Informática na Educação (SBIE 2020)

acumuladas, que lhes confere autonomia para decidir quando, como e o que desejam aprender [Giubilei 1993].

Espera-se que esta pesquisa propicie a criação de um grupo que objetive pesquisar os efeitos cognitivos do Pensamento Computacional sob a população idosa, sendo um tema relevantes para os nossos dias e, de certa maneira, inovador entre os projetos chancelados pela SBC. A estimulação cognitiva na senescência baseada no Pensamento Computacional pode ser um importante instrumento para preservar e até melhorar o desempenho das funções executivas, além de permitir que os idosos estejam socialmente conectados ao mundo moderno.

\section{Referências}

ALVES, Gilda Glauce Martins. Universidade da Terceira Idade como Alternativa de Resgate da Cidadania Idosa: análise do caso da UNIMEP. Dissertação (Mestrado em Educação) Universidade Metodista de Piracicaba, 1997.

ASSIS, Simone A. C. das Neves et al. Efeitos do treino com jogos de videogame na cognição de idosos: revisão sistemática. Scientia Medica, Porto Alegre, v. 25, n. 3, p.1-12, 17 nov. 2015. EDIPUCRS. http://dx.doi.org/10.15448/1980-6108.2015.3.21636.

BRACKMANN, Christian Puhlmann. Desenvolvimento do Pensamento Computacional através de atividades desplugadas na Educação Básica. 2017. 226 f. Tese (Doutorado) Curso de Programa de Pós-graduação em Informática na Educação, Centro Interdisciplinar de Novas Tecnologias na Educação, Universidade Federal do Rio Grande do Sul, Porto Alegre, 2017.

CARDOSO, Nicolas de Oliveira; LANDENBERGER, Thaís; ARGIMON, Irani Iracema de Lima. Jogos Eletrônicos como Instrumentos de Intervenção no Declínio Cognitivo - Uma Revisão Sistemática. Revista de Psicologia da IMED, Passo Fundo, v. 9, n. 1, p. 119-139, nov. 2017. ISSN 2175-5027. https://doi.org/10.18256/2175-5027.2017.v9i1.1941.

FIELD, Andy. Descobrindo a estatística usando o SPSS. 2. ed. Porto Alegre: Bookman, 2009. $684 \mathrm{p}$.

GONZÁLES, Marcos Román. Codigoalfabetización y Pensamiento Computacional en Educación Primaria y Secundaria: validación de un instrumento y evaluación de programas. 226 f. Tese (Doutorado) - Curso de Programa de Doctorado En Educación, Máster Universitario En Innovación e Investigación En Educación, Rererere, 2016.

HINES, William W. et al. Probabilidade e Estatística na Engenharia. 4. ed. Rio de Janeiro: Ltc, 2006.

WING, J. Computational Thinking. 2007. Carnegie Mellon University. Disponível em: <http://www.cs.cmu.edu/afs/cs/usr/wing/www/Computational_Thinking.pdf>.

WING, J. Computational Thinking with Jeannette Wing. Columbia Journalism School, 2014.

CACHIONI, Meire et al. Metodologias e Estratégias Pedagógicas utilizadas por Educadores de uma Universidade Aberta à Terceira Idade. Educação \& Realidade, v. 40, n. 1, p. 81-103, mar. 2015. FapUNIFESP (SciELO). http://dx.doi.org/10.1590/2175-623645741.

BERTOLDI, Josiane Teresinha; BATISTA, Ana Camila; RUZANOWSKY, Samanta. Declínio cognitivo em idosos institucionalizados: revisão de literatura. Cinergis, Santa Cruz do Sul, v. 16, n. 2, p.152-156, abr. 2015. 
IX Congresso Brasileiro de Informática na Educação (CBIE 2020)

Anais do XXXI Simpósio Brasileiro de Informática na Educação (SBIE 2020)

IPEA. Idosos adiam saída do mercado de trabalho e já são 7,8\% dos trabalhadores. 2018. Disponível

em:

<http://www.ipea.gov.br/portal/index.php?option=com_content\&view=article\&id=33628

$\% 3$ Aistoe-online-ipea-idosos-adiam-saida-do-mercado-de-trabalho-e-ja-sao-78-dos-

trabalhadores\&catid=131\%3Asem-categoria\&directory=1\&Itemid=1>

PAOLINI, Karoline Silva. Desafios da inclusão do idoso no mercado de trabalho. Revista Brasileira de Medicina do Trabalho, v. 14, n. 2, p.177-182, 2016. Zeppelini Editorial e Comunicação. http://dx.doi.org/10.5327/z1679-443520162915.

BRANDÃO, Márcia de Chiara e Raquel. Número de lares que dependem da renda de aposentados cresce $12 \%$ em um ano. 2018. Disponível em: $<$ https://economia.estadao.com.br/noticias/geral,numero-de-lares-que-dependem-da-rendade-aposentados-cresce-12-em-um-ano,70002402366>

CAVALLI, Adriana Schüler et al. Estimulação cognitiva para melhoria das funções cerebrais de idosos. Extensio: Revista Eletrônica de Extensão, Florianópolis, v. 14, n. 27, p.101-106, 20 dez. 2017. Universidade Federal de Santa Catarina (UFSC). http://dx.doi.org/10.5007/807-0221.2017v14n27p101.

SCHAIE, K. Warner; WILLIS, Sherry L.. Can decline in adult intellectual functioning be reversed? Developmental Psychology, v. 22, n. 2, p.223-232, 1986. American Psychological Association (APA). http://dx.doi.org/10.1037/0012-1649.22.2.223.

BELL, T.; WHITTEN, I.; FELLOWS, M. Computer Science Unplugged (Ensinando Ciência da Computação sem o uso do computador) Universidade de Canterbury, Nova Zelândia, 2011

SANTOS, Mariana Teles. Desenvolvimento de um programa de intervenção cognitiva para idosos. 2015. 226 f. Tese (Doutorado) - Curso de Programa de Pós-graduação em Psicologia, Universidade Federal de Minas Gerais, Belo Horizonte, 2015.

MARTINEZ, Mariano Sanchez. La Semántica de la Terminología en la Educación de los Mayores. La Gerontagogia. In: I JORNADA SOBRE PERSONAS MAYO-RES Y EDUCADORES SOCIALES. Grupo Editorial Universitario: 1998.

GIUBILEI, Sônia. Uma Pedagogia para o Idoso. A Terceira Idade, Ano V, n. 7, p. 7-14, jun. 1993. 